\title{
Implementation of Gesture Control Robotic Arm for Automation of Industrial Application
}

\author{
Shriya A. Hande ${ }^{1}$, Nitin R. Chopde ${ }^{2}$
}

${ }^{1}$ M.Tech Scholar, Department of Computer Science and Engineering, G.H. Raisoni University Amravati, Maharashtra, India

${ }^{2}$ Professor, Department of Computer Science and Engineering, G.H. Raisoni University Amravati, Maharashtra, India

\section{Article Info}

Volume 7, Issue 4

Page Number: 147-156

Publication Issue :

July-August-2020

\section{Article History}

Accepted : 20 July 2020

Published : 30 July 2020

\begin{abstract}
In today's world, in almost all sectors, most of the work is done by robots or robotic arm having different number of degree of freedoms (DOF's) as per the requirement. This project deals with the Design and Implementation of a "Wireless Gesture Controlled Robotic Arm with Vision". The system design is divided into 3 parts namely: Accelerometer Part, Robotic Arm and Platform. It is fundamentally an Accelerometer based framework which controls a Robotic Arm remotely utilizing a, little and minimal effort, 3-pivot (DOF's) accelerometer by means of RF signals. The Robotic Arm is mounted over a versatile stage which is likewise controlled remotely by another accelerometer. One accelerometer is mounted/joined on the human hand, catching its conduct (motions and stances) and hence the mechanical arm moves in like manner and the other accelerometer is mounted on any of the leg of the client/administrator, catching its motions and stances and in this way the stage moves as needs be. In a nutshell, the robotic arm and platform is synchronised with the gestures and postures of the hand and leg of the user / operator, respectively. The different motions performed by robotic arm are: PICK and PLACE / DROP, RAISING and LOWERING the objects. Also, the motions performed by the platform are: FORWARD, BACKWARD, RIGHT and LEFT. Keywords- DOF, Robotic Arm.
\end{abstract}

\section{INTRODUCTION}

An automated arm is a robot controller, which can perform comparative capacities to a human arm. Automated arms are the crucial piece of practically all the enterprises. In businesses, an automated arm perform different various undertakings, for example, welding, cutting, picking and putting and so forth. Besides the greatest favorable position of these arms is that it can work in unsafe regions and furthermore in the territories which can't be gotten to by human. For instance in NASA's strategic Mars, the Spirit and

Copyright: $(\mathcal{C}$ the author(s), publisher and licensee Technoscience Academy. This is an open-access article distributed under the terms of the Creative Commons Attribution Non-Commercial License, which permits unrestricted noncommercial use, distribution, and reproduction in any medium, provided the original work is properly cited 
Opportunity ramble. It is additionally used to execute exceptionally exact clinical medications and so on. Numerous variations of these robots/mechanical are accessible or planned according to the prerequisite. Scarcely any variations are Keypad Controlled, Voice Control, Gesture Control, and so on. Notwithstanding, a large portion of the mechanical robots are still modified utilizing the run of the mill showing process which is as yet a monotonous and tedious undertaking that requires specialized mastery. Subsequently, there is a requirement for new and simpler ways for programming the robots. In this venture, the signal based framework (utilizing Accelerometer) has been joined to control the mechanical arm just as its foundation utilizing two, little and minimal effort, 3-pivot accelerometers. The prime point of the plan is that the robot and stage begins the development when the administrator makes a motion or act or any movement. The Robotic arm is synchronized with the motions (hand stances) of the administrator and the stage part is synchronized with the motions (leg stances) of the administrator. The objective of this venture is to create systems that help clients to control and program a robot, with a significant level of deliberation from the robot explicit language for example to disentangle the robot programming. These days, mechanical technology arm getting one of the most progressive in the field of innovation. A Robot is an electro-mechanical framework that is worked by a PC program. Robots can be self-ruling or semi-selfruling. A self-governing robot isn't constrained by human and follows up on its own choice by detecting its condition. Greater part of the mechanical robots are independent as they are required to work at fast and with extraordinary precision. In any case, a few applications require semi-self-ruling or human controlled robots. The absolute most usually utilized control frameworks are voice acknowledgment, material or contact controlled and movement controlled. A Gesture Controlled robot is a kind of robot which can be controlled by your hand gestures not by old buttons. You just need to wear a small transmitting device in your hand which included an acceleration meter. This will transmit an appropriate command to the robot so that it can do whatever we want. The transmitting device included a ADC for analog to digital conversion and an encoder, which is use to encode the four bit data and then it will transmit by an RF Transmitter module. At the receiving end an RF Receiver module receive's the encoded data and decode it by and decoder.

This data is then processed by a microcontroller and finally our motor driver to control the motor's. Now its time to break the task in different module's to make the task easy and simple any project become easy or error free if it is done in different modules. As our project is already divided into two different part transmitter and receiver. The applications of robotics mainly involve in automobiles, medical, construction, defense and also used as a fire fighting robot to help the people from the fire accident. But, controlling the robot with a remote or a switch is quite complicated. So, a new project is developed that is, an accelerometer based gesture control robot. The main goal of this project is to control the movement of the robot with hand gesture using accelerometer. The robot is usually an electro-mechanical machine that can perform tasks automatically. Some robots require some degree of guidance, which may be done using a remote control or with a computer interface. Robots can be autonomous, semi-autonomous or remotely controlled. Robots have evolved so much and are capable of mimicking humans that they seem to have a mind of their own.

\subsection{Problem statement}

The traditional wired buttons controlled robot becomes very bulgy and it also limits the distance the robot goes. The Wireless Hand controlled Robot will function by a wearable hand glove from which the 
movements of the hand can be used as the input for the movement of the robot. The basic idea of our project is to develop a system (Robot) which can recognize the Human Interaction with it to accomplish the certain tasks assigned to it. In our project we will design a wearable Hand Glove which will contain the sensors mounted on it to capture the movement of the hand and convert the raw mechanical data into electrical form. This data will be further processed and converted into an understandable format for the lilypad mounted on the Glove. This lilypad will act as a transmitter of the data for wireless communication purpose. Once the transmitted data is received by the receiver module which will be connected to the Microcontroller, it will be processed and further sent to the Microcontroller. Microcontroller will deduce the commands and accordingly it will actuate the motor drivers to control the Motors for various tasks on the robot.

\section{Problem statement}

The traditional wired buttons controlled robot becomes very bulgy and it also limits the distance the robot goes. The Wireless Hand controlled Robot will function by a wearable hand glove from which the movements of the hand can be used as the input for the movement of the robot. The basic idea of our project is to develop a system (Robot) which can recognize the Human Interaction with it to accomplish the certain tasks assigned to it. In our project we will design a wearable Hand Glove which will contain the sensors mounted on it to capture the movement of the hand and convert the raw mechanical data into electrical form. This data will be further processed and converted into an understandable format for the lilypad mounted on the Glove. This lilypad will act as a transmitter of the data for wireless communication purpose. Once the transmitted data is received by the receiver module which will be connected to the Microcontroller, it will be processed and further sent to the Microcontroller. Microcontroller will deduce the commands and accordingly it will actuate the motor drivers to control the Motors for various tasks on the robot.

\section{LITERATURE REVIEW}

In [4], a multimodal interface is combining head motion, speech recognition and tongue motion to control a computer. The combination of sEMG, eyetacking and EEG is proposed in to manipulate a prosthesis with high dexterirty. Such approaches are deemed to provide many more degrees of freedom (DoF) then unimodal control systems, opening up the possibility of harnessing more potential control strategies better suited to each individual user.

Hand gesture recognition using image processing algorithms many times involve use of colour gloves. By tracking this colour glove different hand gestures can be interpreted as described by Luigi Lamberti1 and Francesco Camastra in their paper [7]. Here they have modelled a colour classifier performed by learning vector Quantization. In Paper [4] pattern recognizing algorithm has been used to study the features of hand. There are many Papers where training of hands using a large database of near about 5000-10000 positive and negative images are considered. But this procedure is very tiring and time taking.

Usability is the main gauge of a control system's successfulness. In addition to low-power, convenience and flexibility concerns that has prevailed in the hardware architecture design phase, ease of use, intuitiveness and efficiency are among the key elements that were considered when designing the architecture and processing units of this BoMI. 
For safety issues, latency is very important as well, and should not exceed 300-ms for real time operation as recommended in [3]. The next subsections describe the software elements of the proposed controller, from the sensor firmware to the data fusion and control algorithms

There has been many research works in the field of Hand Gesture based Human Computer Interaction following different algorithms to develop a fast and reliable procedure for gesture recognition. In Paper [1] a three axis accelerometer has been used to read different types of Hand gestures. A combination of accelerometer and gyroscope and the reading are taken in to for analyzing the gesture. Here accelerometer is dedicated for collecting translational dynamic and static change in positional vector of hand and infer it to the movement of mouse whereas gyroscope has been used for rotation of virtual object. There are many papers where gestures are being analyzed using colour gloves [3]. A data glove is a type of glove that contains fiber optics sensor or flex sensors embedded in it to recognize the finger movements.

For Hand gesture recognition, some researchers have tried to perform the early segmentation process using skin colour histogram [6] used overlapping subwindows which is useful to extract invariants for gesture recognition, and distinguish them with a local orientation histogram attribute description indicating the distance from the canonical orientation. This makes the process relatively robust to noise, however, much more time consuming indeed. Defined seven different stages of hand gesture recognition. It includes position of the figure-tip. This is not practically realistic when we have only pointing gestures, but also several gestures, like grasping [2].

\section{METHODOLOGY}

\section{Software Architecture}

Usability is the main gauge of a control system's successfulness. In addition to low-power, convenience and flexibility concerns that has prevailed in the hardware architecture design phase, ease of use, intuitiveness and efficiency are among the key elements that were considered when designing the architecture and processing units of this BoMI.

1.1 Sensor Nodes Firmware Architecture: Sensor nodes must minimize power consumption while guaranteeing adequate motion sampling rate for supporting robust and precise control schemes.

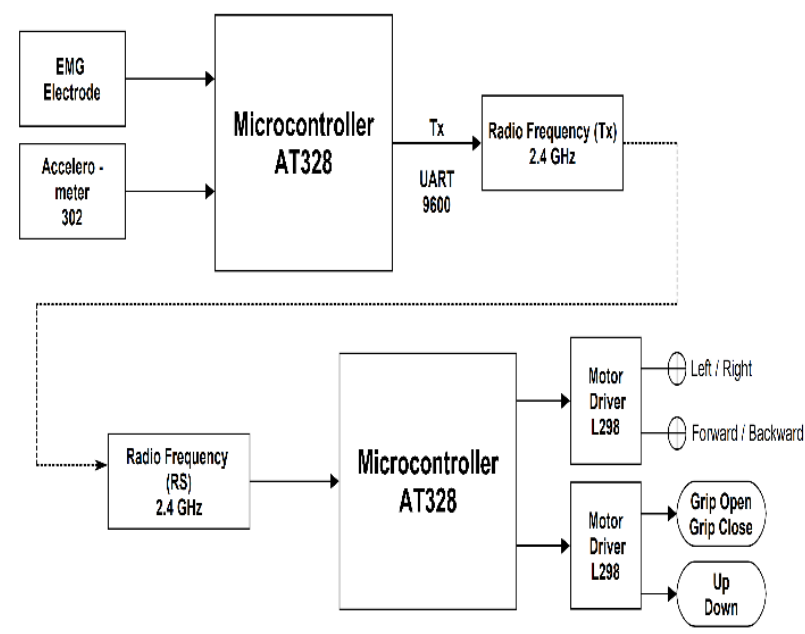

Fig 1. Block diagram of Proposed System

\subsection{Wireless Sensor Network \& Power} Management: As mentioned in Section III-A, the nRF24L01+ is used for the wireless data link of the proposed controller. A star topology is used and the base-station receives the stream of measurement data sent by all the network's peripheral nodes (sensors + Safety-key).

1.3 Data Processing: All the data processing is done on the host platform. Voluntary muscle contractions are read from raw muscle activity signals 
when sEMG features are used whereas motion is sensed from IMU data.

\section{Robotic Arm}

This is the vital part of the system as it is this part which does the Pick and Drop task of the project. Both the Arm and Gripper are equipped with Servo Motor to control the movement. These movements are synchronised with the hand gestures of the user, operating the Robotic Arm.

\section{Camera}

The system uses a smartphone with camera for continuous real time video streaming of the system and its surroundings. An IP-based Android application [13], running on the smartphone enables the system to transmit the real time video wirelessly.

\section{Transmitter Section}

It consists of

1) Acceleromitter
2) flex Sensor,
3) Ardino,
4) Redio frequency modules,
5) LED lights.

Tansmitter section transmit data to the receiver side. Here Acceleromitter and flex sensor are used as a sensors in this project where as Acceleromitter placed on plam of hand gloves and flex sensor are placed on top of fingers of hand gloves.

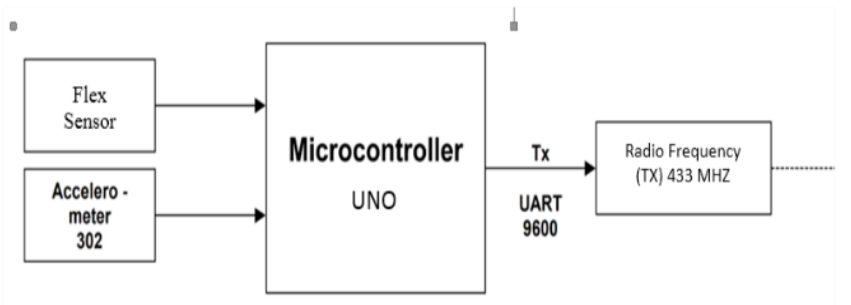

Fig. 2 Block diagram of transmitter section

\section{Accelerometer}

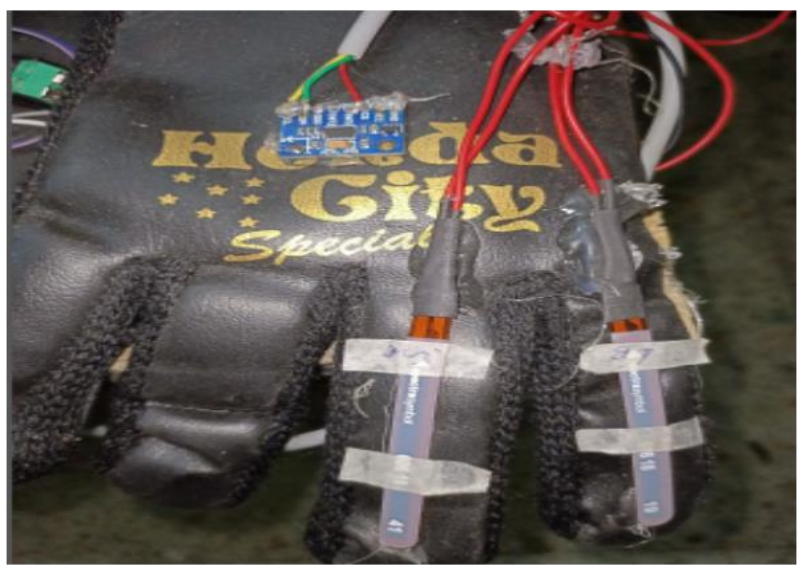

Fig. 3 ADXL302 Accelerometer

Most of the robotic arm is controlled by using accelerometer sensor with an artificial intelligent algorithm. This paper is proposed a gesture recognition based 6DOF robotic arm controller using accelerometer to improve the stability and to detect the rotational gesture of human arm. The arm also has the capability to grab object. To find out the angular position of an object, it is easiest way to fuse 3axis accelerometer and 3axis gyro-meter sensor. A low cost MEMs chip (integrated 3-axis accelerometer) used to detect human arm gesture as well as its angular position. Here gives gesture orientation data to determine dynamic gesture behavior. An artificial algorithm used to evaluate all gesture data which helps to train the robotic arm. The most popular Kalman filter used to find out the exact position of human arm more accurately. The communication between human hand and robotic arm interaction has been established wirelessly over IEEE standard Zigbee protocol interface. The result is that the arm's movement is synchronous with human arm gesture i.e. like a shadow mode. The artificial arm response time is very fast with human arm gesture. The control strategy is easier than other systems like joystick control and this system applicable for industrial purposes. This robotic arm has been developed in Arduino IDE platform and it is also applicable in 
different platform like embedded, intelligent peripheral and so on. In conclusion, some tests has been performed with this robotic arm and the results are discussed.

\section{Micro controller}

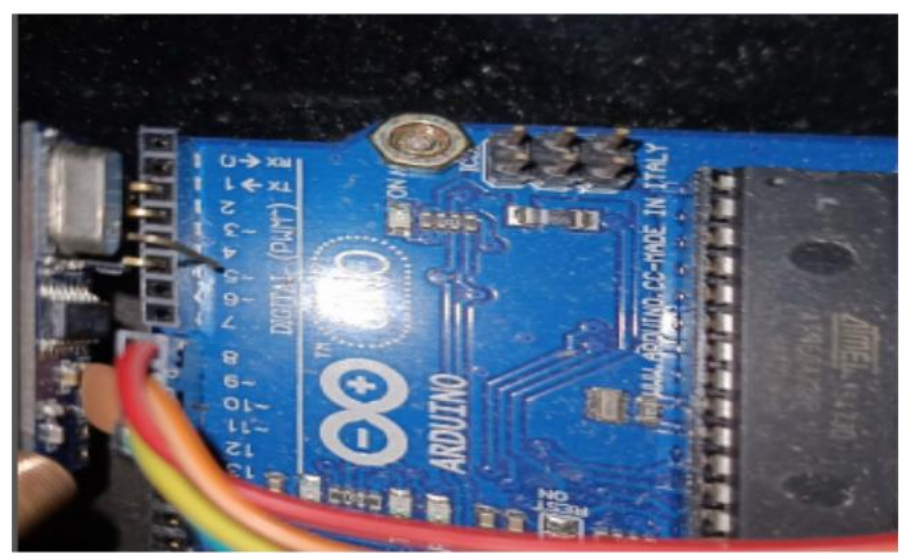

Fig. 4 Arduino Micro is a microcontroller board

The Arduino Micro is a microcontroller board based on the UNO (datasheet), developed in conjunction with Adafruit. It has 20 digital input/output pins (of which 7 can be used as PWM outputs and 12 as analog inputs), a $16 \mathrm{MHz}$ crystal oscill A flex, a micro USB connection, an ICSP header, and a reset button. Uno has 20 digital I/O pins, 6 of which are also analog input pins. Leo has 23 digital I/O pins, 12 of which are also analog input pins. Uno has 3 digital pins on the ICSP header which are repeats of pins on the digital header, pins 11, 12, and 13.

Arduino is an open-source electronics platform based on easy-to-use hardware and software. Arduino boards are able to read inputs - light on a sensor, a finger on a button, or a Twitter message - and turn it into an output - activating a motor, turning on an LED, publishing something online.

The Arduino Uno is a microcontroller board based on the Uno (datasheet). It has 14 digital input/output pins (of which 6 can be used as PWM outputs), 6 analog inputs, a $16 \mathrm{MHz}$ ceramic resonator, a USB connection, a power jack, an ICSP header, and a reset button.

In fact, you already are; the Arduino language is merely a set of $\mathrm{C} / \mathrm{C}++$ functions that can be called from your code. Your sketch undergoes minor changes (e.g. automatic generation of function prototypes) and then is passed directly to a $\mathrm{C} / \mathrm{C}++$ compiler (avr-g++).

\section{Flex Sensor}

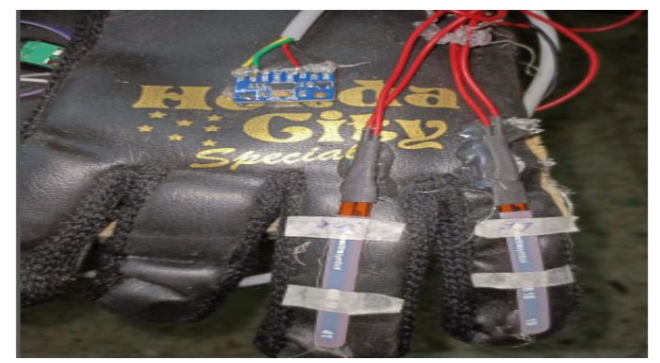

Fig. 5 flex sensor

sensor or bend sensor is a sensor that measures the amount of deflection or bending. Usually, the sensor is stuck to the surface, and resistance of sensor element is varied by bending the surface. As a variable printed resistor, the Flex Sensor achieves great form-factor on a thin flexible substrate. When the substrate is bent, the sensor produces a resistance output correlated to the bend radius-the smaller the radius, the higher the resistance value. Applications. Flex sensor is used in wide areas of research from computer interfaces, rehabilitation, security systems and even music interfaces. It is also famous among students and Hobbyists. The Flex Sensor is a unique component that changes resistance when bent. An unflexed sensor has a nominal resistance of $10,000 \mathrm{ohms}(10 \mathrm{~K})$. As the flex sensor is bent the resistance gradually increases. When the sensor is bent at 90 degress its resistance will range between $30-40 \mathrm{~K}$ ohms. 


\section{Servo motor works}

\section{Redio frequncy Models}

The number of cycles, or times that a wave repeats in a second, is called frequency. Frequency is measured in the unit hertz $(\mathrm{Hz})$, referring to a number of cycles per second. One thousand hertz is referred to as a kilohertz (KHz), 1 million hertz as a megahertz $(\mathrm{MHz})$, and 1 billion hertz as a gigahertz (GHz).

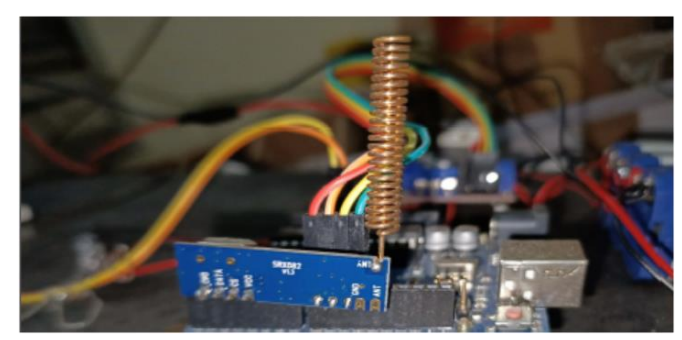

Fig.no. 6 Redio frequency model

\section{BLOCK DIAGRAM OF RECEIVER SECTION}

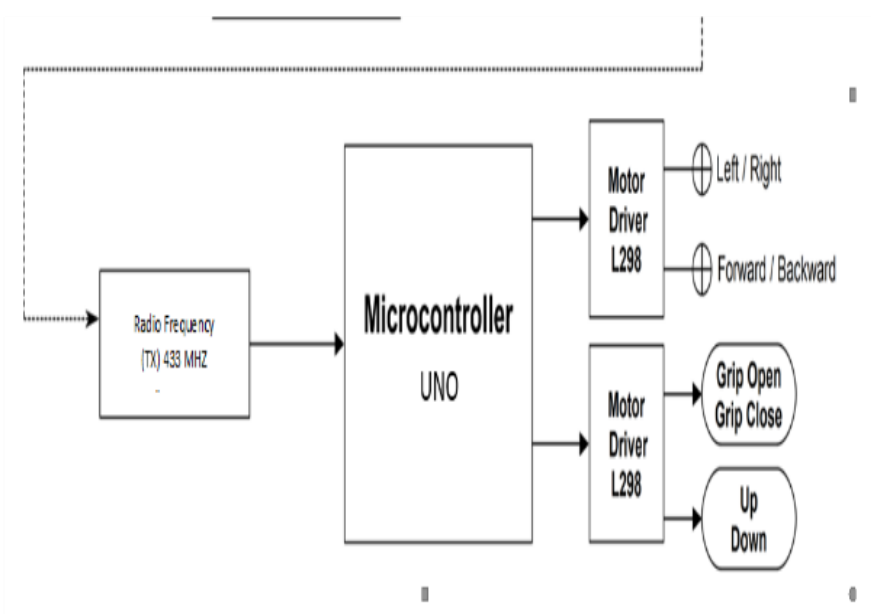

Fig. 7 Block Diagram of Receiver Section

\section{Radio Frequency Models}

The number of cycles, or times that a wave repeats in a second, is called frequency. Frequency is measured in the unit hertz $(\mathrm{Hz})$, referring to a number of cycles per second. One thousand hertz is referred to as a kilohertz $(\mathrm{KHz}), 1$ million hertz as a megahertz $(\mathrm{MHz})$, and 1 billion hertz as a gigahertz $(\mathrm{GHz})$.

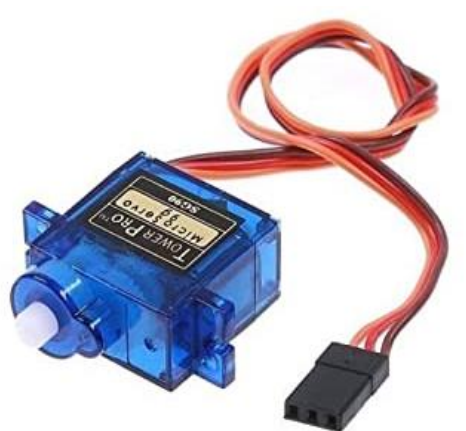

Fig. 8 Servo motor

Servo motor works on the PWM (Pulse Width Modulation) principle, which means its angle of rotation, is controlled by the duration of pulse applied to its control PIN. Basically servo motor is made up of DC motor which is controlled by a variable resistor (potentiometer) and some gears. Servos are used in radio-controlled airplanes to position control surfaces like elevators, rudders, walking a robot, or operating grippers. Servo motors are small, have built-in control circuitry and have good power for their size.

\section{APPLICATIONS \& FUTURE SCOPE}

\section{Application}

- Entertainment applications - Most videogames today are played either on game consoles, arcade units or PCs, and all require a combination of input devices. Gesture recognition can be used to truly immerse a player in the game world like never before.

- Automation systems - In homes, offices, transport vehicles and more, gesture recognition can be incorporated to greatly increase usability and reduce the resources necessary to create primary or secondary input systems like remote controls, car entertainment systems with buttons or similar. 
- An easier life for the disabled - One of the biggest challenges faced today is providing separate and equally non cumbersome services to the differently abled and handicapped. While there are special provisions around the world, there's still huge room for improvement to bring all lives on equal footing. Gesture recognition technology can eliminate a lot of manual labor and make life much easier for those who aren't as fortunate as most of us are.

\section{Future scope}

The project presents a Gesture Control Robotic Arm Using Flex Sensor with seven degrees of freedom. The robotic arm was made of low cost materials that were readily available. The model of the robotic arm was constructed and the functionality was tested. The robotic arm can be controlled over the internet by using Ethernet connectivity and a camera for visual feedback.

Gesture based interfaces allow human computer interaction to be in a natural as well as intuitive manner. This project discussed hardware and software co-design of robotic arm controller using DC motors employing microcontroller ATMEGA16. The robotic hand has been designed to meet all of the original specifications of the project. The fingers are allowing for full motion of the hand. Observations show that the project produces the required motion of the fingers. Such type of hand gesture controlled robotic arm is mostly useful for Industrial, Medical \& Military applications. This type of the hand gesture technology can be used where the humans are unable to sustain in the difficult or harsh environments. This might reduce some of the labor that is used in industry and also the life risk factor.

\section{RESULT}
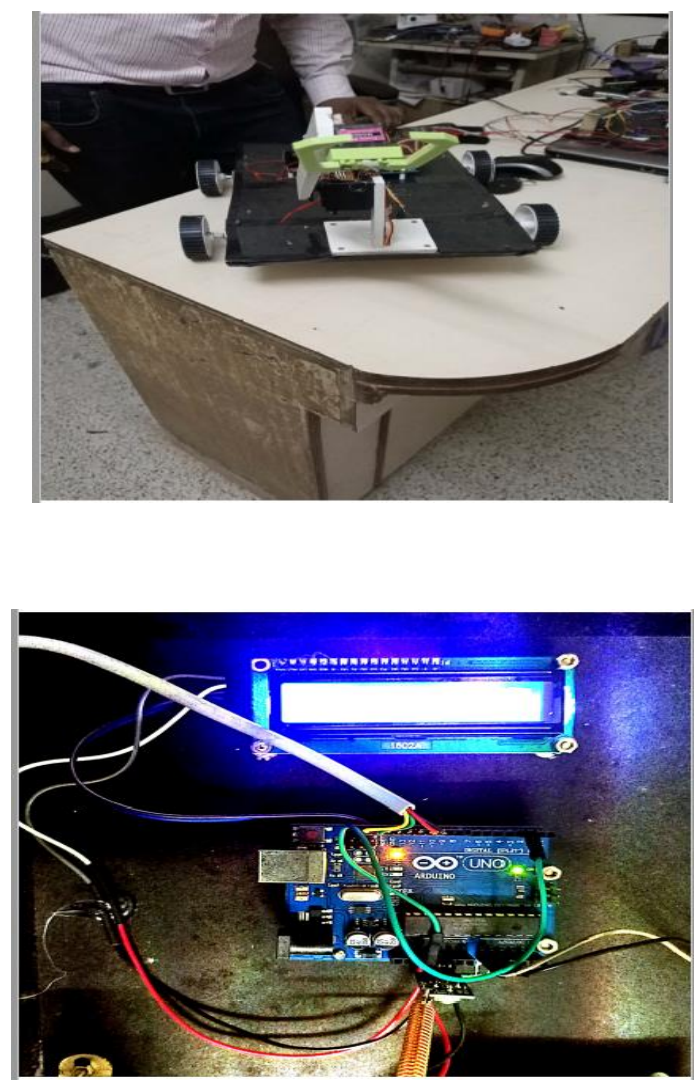

Fig. 9 Grip open

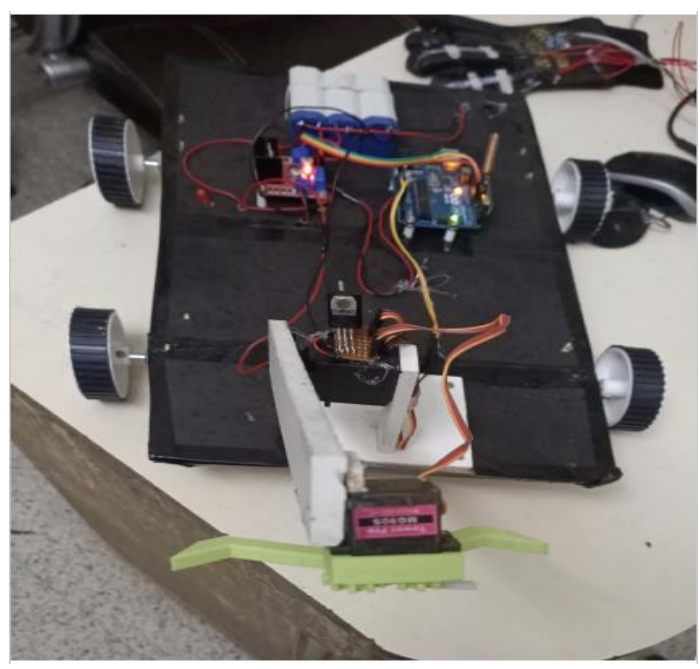




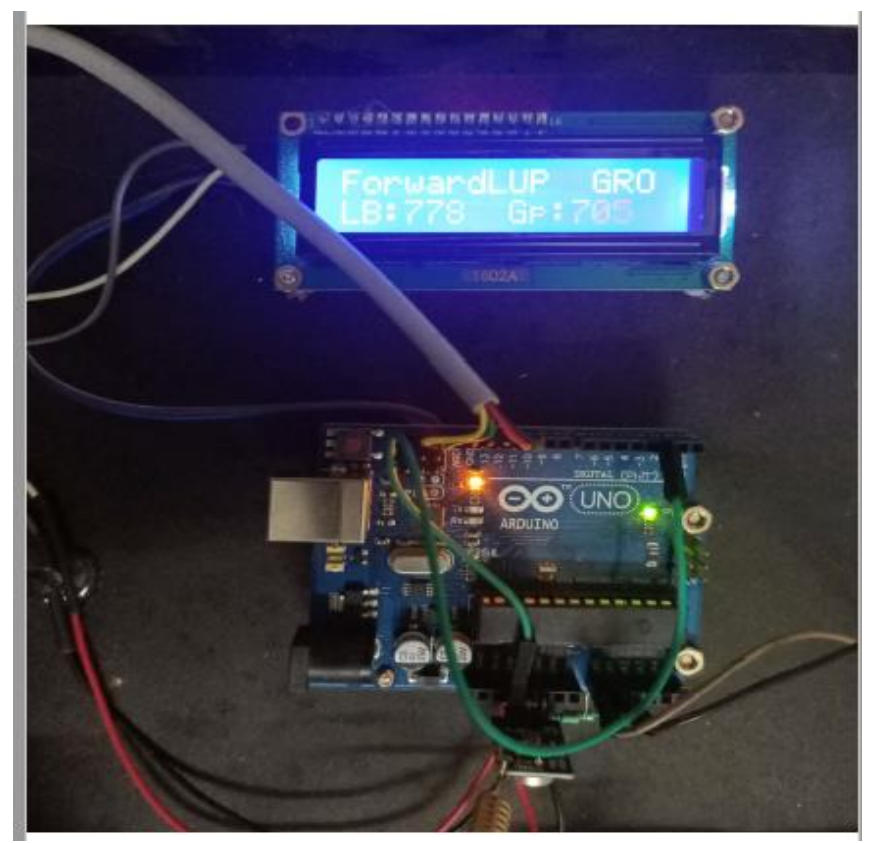

Fig. 10 Grip close

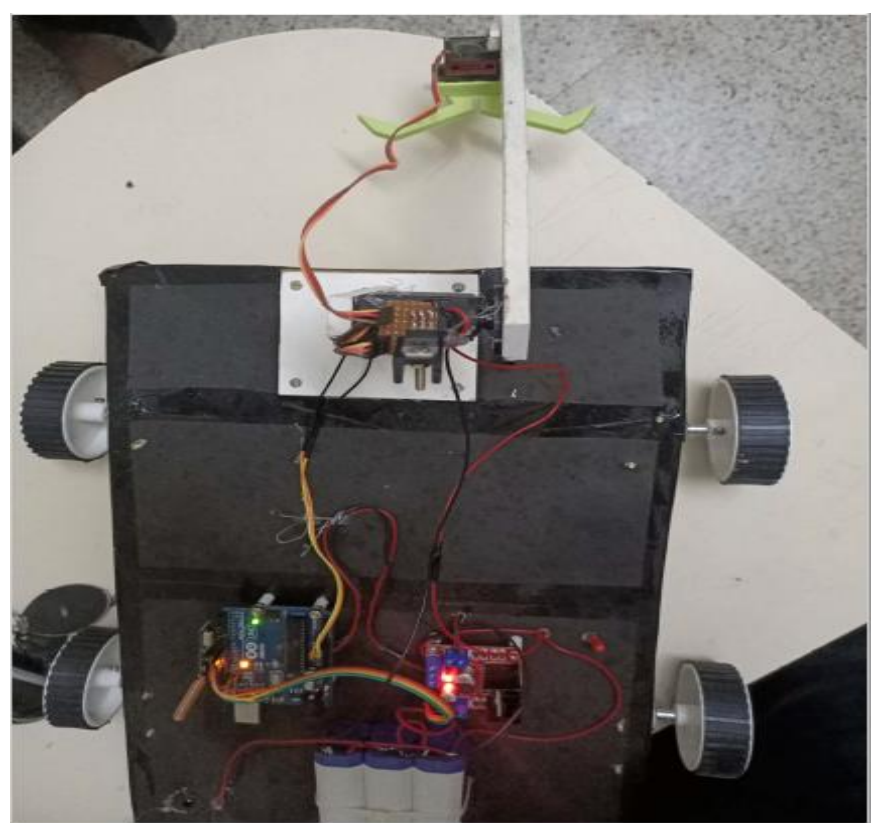

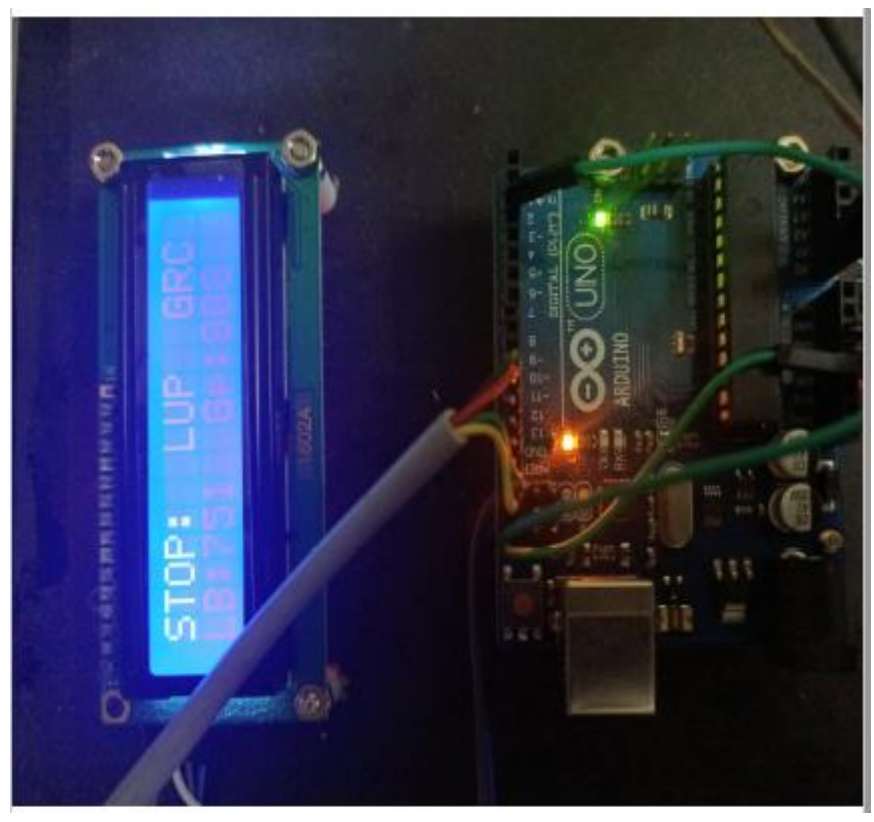

Fig. 11 Elbow up

\section{CONCLUSION}

The project presents a learning Gesture Control Robotic Arm Using Flex Sensor with seven degrees of freedom. The robotic arm was made of low cost materials that were readily available. The model of the robotic arm was constructed and the functionality was tested. The robotic arm can be controlled over the internet by using Ethernet connectivity and a camera for visual feedback.Gesture based interfaces allow human computer interaction to be in a natural as well as intuitive manner. This project discussed hardware and software co-design of robotic arm controller using DC motors employing microcontroller ATMEGA16. The mechanical hand has been intended to meet the entirety of the first particulars of the task. The fingers are taking into consideration full movement of the hand. Perceptions show that the venture creates the necessary movement of the fingers. Such sort of hand motion controlled automated arm is generally helpful for Industrial, Medical and Military applications. This kind of the hand motion innovation can be utilized where the people can't support in the troublesome or 
brutal situations. This may diminish a portion of the work that is utilized in industry and furthermore the existence hazard factor.

\section{REFERENCES}

[1] M. A. Jose and R. de Deus Lopes, "Humancomputer interface controlled by the lip," IEEE J. Biomed. Health Inf., vol. 19, no. 1, pp. 302308, Jan. 2015.

[2] C. L. Fall et al., "Wireless sEMG-based bodymachine interface for assistive technology devices," IEEE J. Biomed. Health Informat., vol. 21, no. 4, pp. 967-977, Jul. 2017.

[3] U. C^ot'e-Allard, C. L. Fall, A. CampeauLecours, C. Gosselin, F. Laviolette, and B. Gosselin, "Transfer learning for sEMG hand gestures recognition using convolutional neural networks," in Proc. 2017 IEEE Int. Conf. Syst., Man,Cybern., 2017, pp. 1663-1668.

[4] H. Park et al., "A wireless magnetoresistive sensing system for an intraoral tonguecomputer interface," IEEE Trans. Biomed. Circuits Syst., vol. 6, no. 6, pp. 571-585, Dec. 2012.

[5] L. N. A. Struijk, L. L. Egsgaard, R. Lontis, M. Gaihede, and B. Bentsen, "Wireless intraoral tongue control of an assistive robotic arm for individuals with tetraplegia," J. NeuroEng. Rehab., vol. 14, no. 1, p. 110, 2017

[6] M. N. Sahadat, A. Alreja, and M. Ghovanloo, "Simultaneous multimodal PC access for people with disabilities by integrating head tracking, speech recognition, and tongue motion," IEEE Trans. Biomed. Circuits Syst., vol. 12, no. 1, pp.192-201, Feb. 2018.

[7] B. Hudgins, P. Parker, and R. N. Scott, "A new strategy for multifunction myoelectric control," IEEE Trans. Biomed. Eng., vol. 40, no. 1, pp. 82-94, Jan. 1993.
[8] Pedro Neto, J. Norberto Pires, A. Paulo Moreira, "Accelerometer-Based Control of an Industrial Robotic Arm" Available at: http://arxiv.org/ftp/arxiv/projects/1309/1309.20 90.pdf

[9] Dr. R. V. Dharaskar, S. A. Chhabria, Sandeep Ganorkar, "Robotic Arm Control Using Gesture and Voice", In International Journal of Computer, Information Technology \& Bioinformatics (IJCITB), Vol. 1, Issue 1, pp. 4146 Available at: http://www.ijcitb.com/issues/project_9.pdf

[10] S. Waldherr, R. Romero and S. Thrun, 2000, "A gesture based interface for human-robot interaction", In Autonomous Robots in Springer, vol. 9, Issue 2, pp. 151-173.

\section{Cite this article as :}

Shriya A. Hande, Nitin R. Chopde, "Implementation of Gesture Control Robotic Arm for Automation of Industrial Application", International Journal of Scientific Research in Science and Technology (IJSRST), Online ISSN : 2395-602X, Print ISSN : 23956011, Volume 7 Issue 4, pp. 147-156, July-August 2020. Available at doi : https://doi.org/10.32628/IJSRST207442 Journal URL : http://ijsrst.com/IJSRST207442 